Tresselt, \& Helfer, 1967b), all $10 \mathrm{Ss}$ reported seeing one of two organizations, which reverse back and forth, seemingly spontaneously, as in Necker cube reversals, during the 120 -sec observation period employed with each of the five display sequences shown in Table 1.

In one organization, Ss report, for example, with a line of 20 "Os," a line of 20 "Os" with a slight flicker present, but which may suddenly change to a second organization of from only five to six "Os" spaced equally apart across the width of the display surface (i.e., $20 \mathrm{~cm}$ ) and moving from left to right, just as "ducks in a shooting gallery." If, for the 20 "Os," we substitute any one of the other content lines shown in Table 1 , all $10 \mathrm{Ss}$ again report seeing either all 20 characters with a slight flicker or seeing a sudden change to the "ducks in the shooting gallery" type of movement, but with the different content inputs (i.e., "O-" or "O- $/$," etc.) that are moving from left to right most remarkably all occupying the identical spatial location. Thus, with the input array "O-/!," all Ss report that it appears as if the letter " $O$ " had a dash, a slash, and an exclamation point inside it. The dash and exclamation point alone appears like a plus sign moving across the display surface. These novel effects, including both apparent movement and spatial displacement, we have called "dynamic sequential displacement."

Each of the 10 Ss observed each of the five different display sequences five times each in a different random order and were asked to report, for each 120-sec observation period, each time the organization of the display sequence changed from 20 characters to 5 or 6 characters in movement, or back again. Time spent in observing each organization also was recorded. Since all Ss were quite consistent across the five different time observation periods used on each of the five different display sequences, the results for all observation periods were combined and averaged. The mean number of reversals per observation period was 4.7 , with a range of from 2 to 11 . Time spent per organization was $51.3 \mathrm{sec}$ on the average for the 20 characters and $68.7 \mathrm{sec}$ on the average for 5 or 6 characters in movement, per observation period. However, the critical finding of the study is not the actual number of reversals that occur or time spent per organization, but rather that two entirely different processings or organizations of the input array are possible and that the visual dynamics of the array are sufficiently unstable so that the system oscillates between these two different organizational states.

One organization loses all temporal ordering information and codes the input array as if all inputs had occurred simultaneously. The other organization, which occurs equally often, codes the input array in such a way that (1) the visual system is detecting only what appears to be 5 or 6 spatially separate inputs rather than 20 inputs, but in apparent movement, and (2) when input content differs, such as "Os," "-s," "/s," and $! s$ " interleaved, these inputs suffer a spatial displacement such that all four inputs appear to be occupying the identical physical location in space, when, in fact, all four inputs are spatially adjacent to one another in space.

Obviously, very complicated visual dynamics must be occurring when the visual system is required to process such a highly complex spatio-temporal input configuration. ${ }^{2}$ In other papers (Mayzner \& Tresselt, in press; Mayzner, Tresselt, \& Helfer, 1967b), we have attempted to construct a visual information-processing model based on excitatory-inhibitory field interactions designed to handle certain sequential blanking and displacement effects, but the "dynamic sequential displacement" effect reported here must await further development of our model before it can begin to account satisfactorily for the present rather complex and novel findings.

\section{REFERENCES}

KAHNEMAN, D. An onset-onset law for one case of apparent motion and metacontrast. Perception \& Psychophysics, 1967, 2, 577-584.

MAYZNER, M. S. The research potential of a computer-based cathode-ray tube display system. Behavior Research Methods \& Instrumentation, 1968, 1, 41-43.

MAYZNER, M. S., \& TRESSELT, M. E. Studies in sequential perception: Looking at one thing but finding another. Perceptual \& Motor Skills, $1966,23,257-258$.

MAYZNER, M. S., \& TRESSELT, M. E. Visual information processing with sequential inputs: A general model for sequential blanking, displacement, and overprinting phenomena. Annals of The New York Academy of Sciences, in press.

MAYZNER, M. S., TRESSELT, M. E., \& HELFER, M. S. A research strategy for studying certain effects of very fast sequential input rates on the visual system. Psychonomic Monograph Supplements, 1967a, 2(5, Whole No. 21), 73-81.

MAYZNER, M. S., TRESSELT, M. E., \& HELFER, M. S. A provisional model of visual information processing with sequential inputs. Psychonomic Monograph Supplements, 1967b, 2(7, Whole No. 23), 91-108.

MAYZNER, M. S. TRESSELT, M. E., ADRIGNOLO, A. J., \& COHEN, A. Further preliminary findings on some effects of very fast sequential input rates on perception. Psychonomic Science, 1967, 7, 281-282.

THORSON, J., LANGE, G. D., \& BIEDERMAN-THORSON, M. Objective measure of the dynamics of a visual movement illusion. Science, $1969,164,1087-1088$.

UTTAL, W. R. Masking of alphabetic character recognition by dynamic visual noise (DVN). Perception \& Psychophysics, 1969, 6, 121-128.

\section{NOTES}

1. This research was supported by Grant No. GB-8037 from the National Science Foundation to the first author.

2. Eye movements would not seem to be involved in "dynamic sequential displacement," since a control display was constructed, which consisted of two lines of characters, as those shown in Table 1, one line directly below the other, but with reversed display orders in the two lines. With such a display, all Ss again report "dynamic sequential displacement," but with movement from left to right in the top line and the opposite movement, i.e., from right to left, in the bottom line, as would be expected if the effect is central in origin.

\title{
Transfer effects of an observation stimulus as a function of its relevancy
}

STANFORD H. SIMON, Veterans
Administration Center, Wood, Wis. 53193

Three groups of Ss, 20 each, learned three successive concept identification (CI) tasks, on which one group had no observation stimulus ( $S^{\circ}$ ), a second group had a relevant $S^{\circ}$, and a third group had an irrelevant $S^{\circ}$. Each type of $S^{\circ}$ continued to affect CI learning as a function of its relevancy. Predictions of relative improved performance on later tasks by the irrelevant $S^{o}$ group, based on a rational operator model, did not occur. 
An earlier study (Simon \& Jackson, 1968) showed that when a dot was used as an observation stimulus $\left(\mathrm{S}^{\circ}\right)$ and was introduced in a concept-identification learning task, it significantly facilitated learning if it was relevant $\left(\mathrm{RS}^{\circ}\right)$, i.e., occurred over the relevant dimension, and significantly retarded learning if it was irrelevant (IS $\left.{ }^{\circ}\right)$, i.e., occurred over an irrelevant dimension. This effect also occurred when the $S^{\circ}$ was introduced for the first time in a second learning task, i.e., after $S$ was familiar with the task (Simon \& Jackson, 1967). What would happen if an So were present on every trial during a series of learning tasks? A group having an RS o should show continuous improvement. If Ss operated rationally, they should be able to achieve one-trial learning by the second or third learning task. A group having an IS $^{\circ}$ should show some improvement on a "learning-to-learn" basis (Simon \& Jackson, 1967). After an $S^{\circ}$ became identified as an $I S^{\circ}$, it would identify one of the irrelevant variables. This should lead to faster learning than in a group with no $\mathrm{S}^{\circ}\left(\mathrm{NS}^{\circ}\right)$ by reducing the number of irrelevant dimensions to be considered. By the second or third learning task, therefore, an IS ${ }^{\circ}$ group should do better than an NS $^{\circ}$ group, but not as well as an $\mathrm{RS}^{\circ}$ group. It was the purpose of this study to assess the effects of an $S^{\circ}$ on repeated learning as a function of the relevancy of the $S^{0}$.

\section{METHOD}

A 11 S s learned three concept-identification tasks presented on three decks of cards. The procedure was identical to that used in the previous studies. Briefly, the first task consisted of two dimensions defined by the letters " $d$ " and "h," with two levels to each dimension, defined as the upper and lower case of the letters. One level of each dimension was presented on each card; the positions of the letters relative to each other were varied systematically. This provided eight combinations, presented in random order in each of four replications for a total of 32 cards.

The second task was similar to the first except that it involved three dimensions defined by the letters "q," "r," and "t." The third task also had three dimensions defined by the letters "g," "n," and "a." Both the second and third tasks had the same two levels to each dimension as the first task and each had 48 cards presented in random order. All cards were shuffled after each $S$ had completed the task.

For all Ss, the relevant dimension in the first task was " $d$," in the second task, "t," and in the third task, "g." In all tasks, S had to identify the cards as belonging to
"Group 1" or "Group 2." Cards with the upper case of the relevant dimension belonged in Group 1, those of the lower case belonged in Group 2. The correct answer was written on the back of each card; $S$ recorded his guess, turned the card, then recorded the correct answer. The Ss were admonished not to turn a card back again while they were taking the task. However, at the end of each of the first two tasks, there was a card with a written explanation of the task solution, with instructions to go back over the task if necessary to check on the solution. This was included to minimize differences related to the possibility of some Ss not knowing the structure of the solution.

As in the previous studies, a dot over a stimulus dimension served as an $\mathrm{S}^{\circ}$. Three groups of $20 \mathrm{Ss}$ each were used. For all three tasks, the $\mathrm{NS}^{\circ}$ group had no $\mathrm{S}^{\mathrm{o}}$, the RS $^{\circ}$ group had a dot over the relevant dimension of each task, and the IS ${ }^{\circ}$ group had a dot over the " $h$ " dimension in the first task, the " $\mathrm{q}$ " dimension in the second task, and the "a" dimension in the third task.

The Ss were introductory psychology students. They were tested in carefully proctored groups of up to nine, with each condition represented equally. The Ss were assigned randomly to the groups.

RESULTS AND DISCUSSION

Since the first learning task had only 32 trials, only the first 32 trials were analyzed for Tasks 2 and 3 . Figure 1 shows the mean errors for all three groups over the three learning tasks.

An analysis of variance for repeated measures found a significant methods

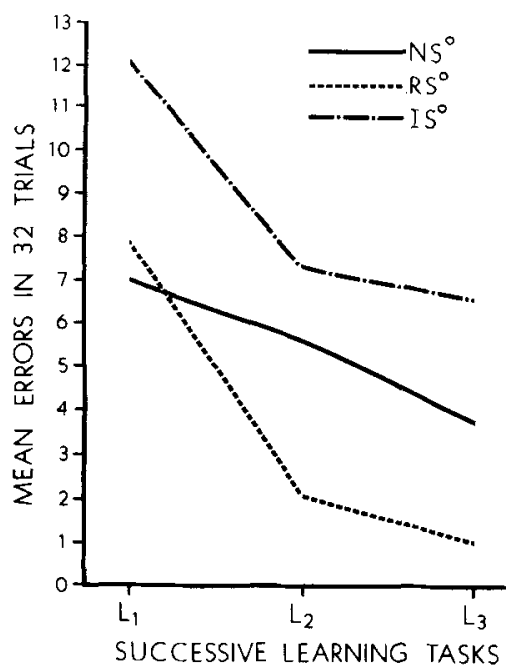

Fig. 1. Mean error scores for groups with no $\left(\mathrm{NS}^{\circ}\right)$, relevant $\left(\mathrm{RS}^{\circ}\right)$, and irrelevant $\left(I^{\circ}\right)$ observation stimuli for three successive learning tasks. effect $[F(2,57)=8.72, p<.001]$ and a significant effect for the different tasks $[F(2,114)=27.35, p<.001]$, showing the increased learning efficiency that took place across tasks; the interaction was not significant. Using the pooled error term and collapsing across tasks, all three groups differed significantly from each other: $\mathrm{NS}^{\circ}$ vs IS ${ }^{\circ}, \mathrm{t}(57)=4.533, \mathrm{p}<.001$; $N^{\circ}{ }^{\circ} R^{\circ}, \quad t(57)=2.62, \quad p<.02$; IS ${ }^{\circ}$ vs $\mathrm{RS}^{\circ}, \mathrm{t}(57)=7.15, \mathrm{p}<.001$. The IS ${ }^{\circ}$ group had the most errors, the RS group had the least errors, and the NSO group was in the middle. Except for the nonsignificant reversal between RS $^{\circ}$ and NS ${ }^{\circ}$ on the first task, the positions of the three curves do not cross each other.

When the groups are collapsed, most of the transfer effects occur between Tasks 1 and 2 . Using a pooled error score, Task 1 vs Task 2 has a $\mathrm{t}(114)=5.9, \mathrm{p}<.001 ;$ Task 2 vs Task 3 has a $t(114)=1.511, p<.1$; and Task 1 vs Task 3 has a $t(114)=6.79$, $\mathrm{p}<.001$.

Figure 1 shows that by the third task, the $\mathrm{RS}^{\circ}$ group averaged 1.15 errors, which is slightly more than would be expected if all Ss operated on a strictly rational basis. Actually, 12 Ss had perfect scores, 5 others made only one error, and 3 had more than one error.

By contrast, the IS $^{\circ}$ group did not seem to use the IS ${ }^{\circ}$ as predicted; that is, they do not do better than the NS ${ }^{\circ}$ group, even by the third task. A rational operator who accepted an IS $^{\circ}$ as an IS ${ }^{\circ}$, and would therefore have to select from only two, rather than three, dimensions, ought to make no more than one error, and there should be as many with one error as no errors. Actually, the $I S^{\circ}$ group, by the third task, had only 2 Ss with no errors, 5 Ss with one error, and 13 with more than one error.

In the NS ${ }^{\circ}$ group, a rational operator should get no more than two errors, with each $1 / 3$ of the Ss expected to obtain zero, one, and two errors. By the third task, there was only one $S$ with zero errors, eight with one error, four Ss with two errors, and seven with more than two errors.

The data shows a strong transfer effect for both the RS $^{\circ}$ and IS $^{\circ}$, although perhaps not for the same reasons. It may be that the IS $^{\circ}$ continues to retard learning because Ss expect that on a new task it will becorne an RS $^{\circ}$, i.e., expect it to be relevant to the learning task.

\section{REFERENCES}

SIMON, S. H., \& JACKSON, B. The effect of the relevancy of an observation stimulus on second learning in concept identification. Psychonomic Science, 1967, 8, 531-532.

SIMON, S. H., \& JACKSON, B. Effect of a relevant versus irrelevant observation stimulus on concept identification learning. Journal of Experimental Psychology, 1968, 76, 125-128. 